\title{
Variation in convective properties across the HR diagram
}

\author{
Joel Tanner, Sarbani Basu, Pierre Demarque and Frank Robinson \\ Yale University, New Haven, Connecticut, USA
}

\begin{abstract}
We perform 3D radiative hydrodynamic simulations to study convection in lowmass main-sequence stars with the aim of improving stellar models. Comparing models from a $0.90 M_{\odot}$ evolutionary track with $3 \mathrm{D}$ simulations reveals distinct differences between simulations and mixing length theory. The simulations show obvious structural differences throughout the superadiabatic layer where convection is inefficient at transporting energy. The discrepancy between MLT and simulation changes as the star evolves and the dynamical effects of turbulence increase. Further, the simulations reveal a T-tau relation that is sensitive to the strength of the turbulence, which is in contrast to $1 \mathrm{D}$ stellar models that use the same T-tau relation across the HR diagram.
\end{abstract}

Keywords. stars: atmospheres, stars: evolution

The convective transport of energy in stars plays an important role in accurately determining stellar structure, particularly in the near-surface layers. Commonly used treatments of convection in stellar modeling such as mixing length theory (MLT) (BöhmVitense, 1958), are a large source of uncertainty, especially when applied to transition regions between radiative and convective energy transport. While MLT works very well in deep layers, it breaks down near the surface where radiation carries a significant fraction of the total energy flux.

A $3 \mathrm{D}$ simulation is characterized by its surface gravity, effective temperature and chemical composition. We get the surface gravity, stellar flux and initial stratification for each simulation from 1D stellar evolution models. The simulation domain is located at the top of the convection zone, with the top and bottom of the domain located at approximately 2 and 9 pressure scale heights above and below the photosphere, respectively. The domain is small enough that curvature and radial variation in gravity can be safely ignored. The vertical walls are periodic and the horizontal walls are free slip and impenetrable (closed box).

The code is a finite-difference code on a staggered grid and simultaneously solves the mass-, momentum-(Navier-Stokes) and energy conservation equations consistently coupled with the radiative transfer equations. The subgrid-scale model employed is the one from Smagorinsky (1963). The radiation flux is computed using the 3D Eddington approximation (Unno \& Spiegel, 1966).

Including turbulence in stellar models alters the structure of the outer layers. We find that $3 \mathrm{D}$ simulations produce turbulent pressure that can be in excess of $10 \%$ of the gas pressure, which is an effect that is not present in MLT. In particular, the superadiabaticity is larger and occurs closer to the surface in turbulent simulations than in static 1D models.

Fig. 1 compares the SAL from a 3D turbulent simulation to the SAL computed with a 1D stellar model. The 1D model underestimates both the the height and breadth of the superadiabatic peak at all stages of evolution. Further, the difference between the MLT and simulated SALs are greater in the more evolved models where the turbulent pressure is larger. 

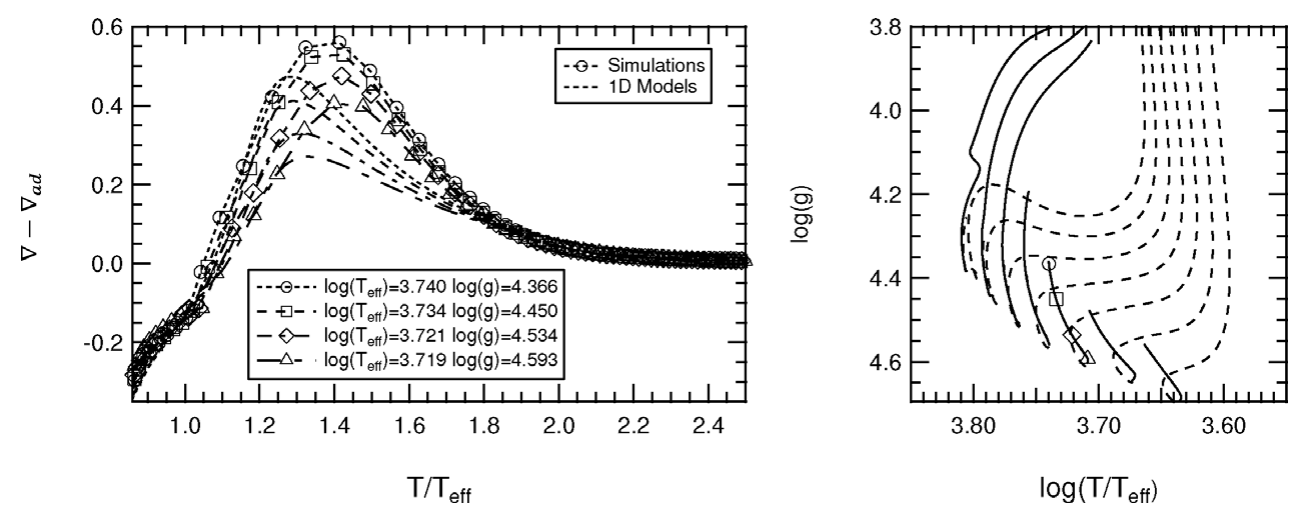

Figure 1. Comparison of the superadiabaticity in 1D models and 3D simulations. The 1D models correspond to different evolutionary states on a $0.90 M_{\odot}$ track. Locations of the $1 \mathrm{D}$ models in the $\log (\mathrm{g})-\log (\mathrm{T})$ are shown in the left panel with pre- and post- main sequence evolutionary tracks for reference.

Fig. 2 shows the turbulent pressure at different stages of evolution. During the evolution, the effective temperature increases slightly, but there is a dramatic decrease in the surface gravity. Both effects contribute to higher turbulent pressure, which reaches nearly $14 \%$ of the gas pressure.

Although convection is driven in the unstable regions, convective overshoot affects the structure above the photosphere. This leads to altered $T-\tau$ relations in the presence of convective overshoot. The right panel of Fig. 2 shows the differences between the 1D Eddington $T-\tau$ and the simulated $T-\tau$ relations. Contrary to what is assumed in $1 \mathrm{D}$ stellar modeling, the simulated $T-\tau$ relations change as the star evolves, and can differ from the Eddington relation by more than $15 \%$.
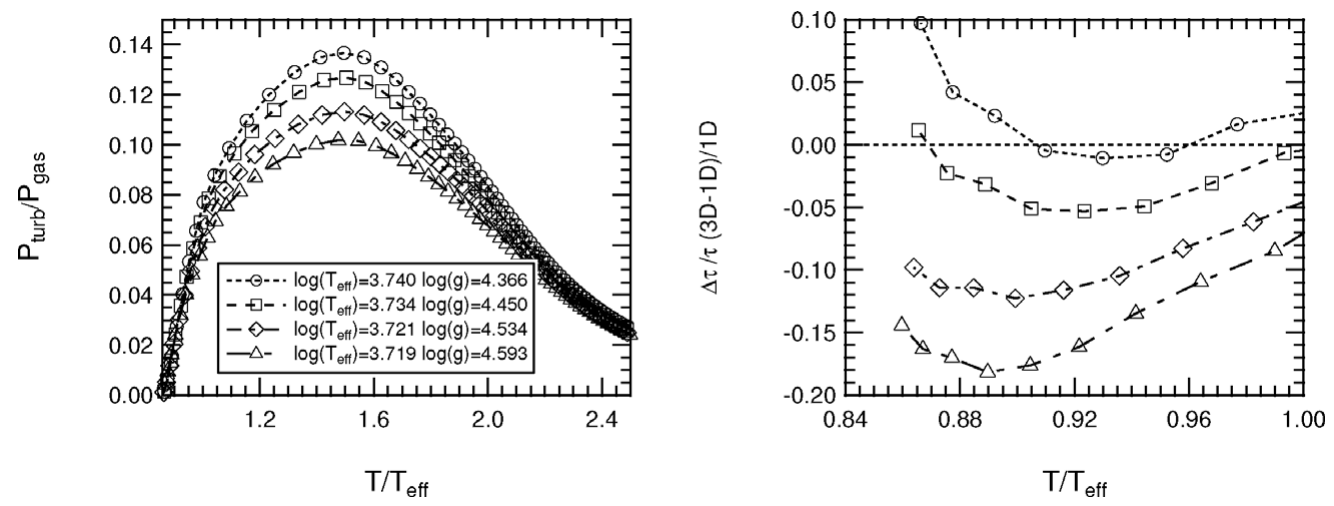

Figure 2. Turbulent pressure in simulations of the SAL for models along a $0.90 M_{\odot}$ track. The turbulence alters the 1D Eddington $T-\tau$ relation as the star evolves. Differences between the simulated and model $T-\tau$ relations are shown in the right panel.

\section{References}

Böhm-Vitense, E., 1958, S. Astrophys., 46, 108

Smagorinsky, J., 1963, Mon. Weather Rev., 91, 99

Unno, W. \& Spiegel, E. A., 1966, PASJ, 18, 85 